\title{
APPLICATIONS OF THE QUARTZ TUNING FORK IN CLASSICAL AND SUPERFLUID HYDRODYNAMICS
}

\begin{abstract}
David SCHMORANZER, Marco LA MANTIA, Ladislav SKRBEK ${ }^{*}$
Abstract: We present a short review of the multitude of applications of the commercially produced quartz tuning fork in cryogenic fluid dynamics, using cold gaseous, normal liquid and superfluid ${ }^{4} \mathrm{He}$ as working fluids with extraordinary, but well-known and tunable physical properties. While the central focus of this work is the use of the tuning fork as a detector of classical and quantum turbulence, we also report other studies and applications, mainly on cavitation, pressure-, thermo- and viscosimetry. Finally, we mention briefly our recent studies of the acoustic emission due to these high-Q oscillators and its effect on the characteristics of their resonance.
\end{abstract}

\section{INTRODUCTION}

While the study of turbulence and fluid dynamics in general has had its place among the most important topics of interest of the scientific community already since the times of Leonardo da Vinci, it was in the last century that this field has seen some remarkable progress. From numerous attempts to develop fundamental theories describing the general properties of turbulence, through the many meticulously performed experiments before the age of computers, to modern and powerful techniques allowing to reconstruct full 3D velocity fields, these advances enable fluid dynamics to remain a steadily evolving and developing field of research. A small, but nonetheless significant part of this progress is owing to the study of various oscillatory flows due to moving submerged bodies, which is a proved and relatively easy means of generating and probing flows under controlled laboratory conditions [1].

Another essential development during the last century that aided this kind of laboratory investigations was the liquefaction of the last remaining noble gas - helium - but perhaps it was one of its amazing consequences that had the bigger impact - the discovery of superfluidity (for a comprehensive review, see, e.g., Ref. [2]). Although for a long time, superfluid dynamics and quantum turbulence [3, 4] have been considered as more or less independent fields separated from mainstream fluid dynamics, it turns out that the use of cryogenic helium in fluid dynamical experiments, both classical and quantum, holds great potential $[5,6,7]$. Due to its extremely low kinematic viscosity [8], normal liquid helium (for historical reasons called $\mathrm{He}$ I) enables studying flows with high Reynolds and Rayleigh numbers literally in tabletop experiments (see, e.g., the turbulent convection studies [9, 10,11], the investigations of von Kármán flow [12], or the measurement of vortex density spectra [13]), thus opening new possibilities in fluid dynamics research without the need to build large very costly facilities that would achieve the same goals with traditional use of air or water as working fluids.

This paper represents a short summary of the latest developments in the study of oscillatory flows in cryogenic helium utilizing a specific and very sensitive tool - the quartz tuning fork. The quartz tuning fork itself is a relatively recent addition to the family of oscillating objects, such as wires [14], grids [15, 16] and spheres $[17,18]$ that are commonly used to study cryogenic flows. It is produced commercially in massive quantities as a frequency standard for digital watches and other electronics (the most common forks resonate at $2^{15} \mathrm{~Hz}$ ) and therefore is available for extremely low prices. The tuning fork is a very sensitive detector (its quality factor $Q$ could exceed $10^{5}$ in ${ }^{4} \mathrm{He}$ at $\mathrm{mK}$ temperatures) capable of generating oscillatory flow on its own or it can be used as a sensing element in externally generated flows [19]. Its behavior and use is thoroughly described in [20,21], including a hydrodynamic model of the fork, therefore, on the following lines, a brief and simplified description will suffice.

\footnotetext{
* Faculty of Mathematics and Physics, Charles University in Prague, V Holesovickach 2, 180 00, Praha 8, Czech Republic e-mail: david.schmoranzer@mff.cuni.cz, lamantia@nbox.troja.mff.cuni.cz, skrbek@nbox.troja.mff.cuni.cz
}

This is an Open Access article distributed under the terms of the Creative Commons Attribution License 2.0, which permits unrestricted use, distribution, and reproduction in any medium, provided the original work is properly cited. 


\section{BASIC TUNING FORK PROPERTIES AND SPECIFICATIONS}

One of the main reasons why the tuning forks have become great experimental tools is the fact that their mechanical and electrical properties are interconnected due to the piezoelectric effect in quartz. Therefore the tuning fork can be described mathematically either as a mechanical oscillator or as an equivalent RLC series resonance circuit [20]. From these descriptions it follows that the force acting on the prongs, $F$, is proportional to the applied driving voltage and the response of the fork, i.e., the passing current, is in turn proportional to the velocity of the tips of the prongs, $U$. Therefore, after calibration, the fork can be used to measure, e.g., the dependence $F=F(U)$ and thus establish the critical velocity for the transition to turbulence. The standard calibration procedure is also described in [20] and a summary of new and more accurate calibration methods is presented in [22]. The geometry and surface roughness of a typical tuning fork is shown in Fig. 1. When operated in its linear regime, the

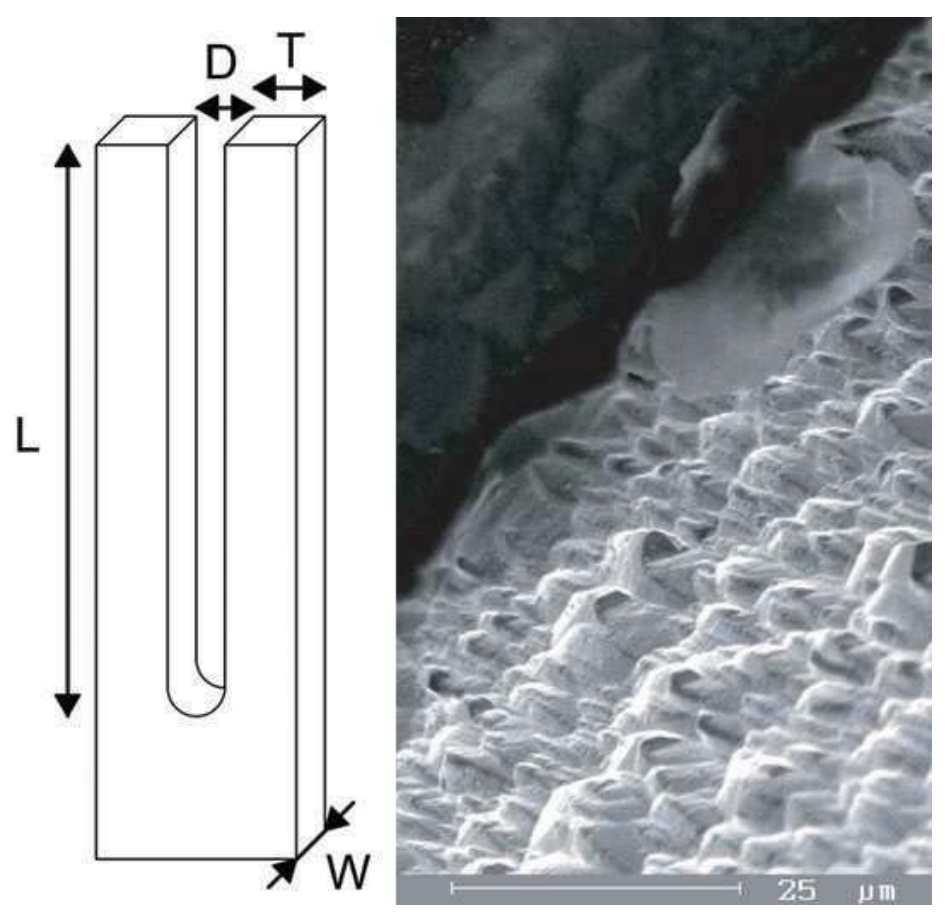

Figure 1: A sketch of the tuning fork with its dimensions marked (left) and a micrograph showing its surface roughness (right).

resonance of a submerged tuning fork is of Lorentzian form and its parameters are described to a good degree of accuracy by the following equations:

$$
\begin{aligned}
\left(\frac{f_{\text {Ovac }}}{f_{0}}\right)^{2} & =1+\frac{\rho}{\rho_{\mathrm{q}}}\left(\beta+B \frac{S}{V} \sqrt{\frac{\eta}{\pi \rho f_{0}}}\right) \\
\Delta f & =\Delta f_{\mathrm{vac}}+\frac{1}{2} \sqrt{\frac{\rho \eta f_{0}}{\pi}} \mathcal{C} S \frac{\left(f_{0} / f_{0 \mathrm{vac}}\right)^{2}}{m_{\mathrm{vac}}}
\end{aligned}
$$

where $f_{0 \mathrm{vac}}$ is the resonant frequency in vacuum, $f_{0}$ is the resonant frequency in the fluid of density $\rho$ and viscosity $\eta$. Next, $\rho_{\mathrm{q}}$ is the density of quartz, $\beta, B, \mathcal{C}$ are fitting parameters and $S=2(T+W) L$ is the surface of a prong of the tuning fork, and $V=T W L$ is its volume. Finally, $\Delta f$ is the resonant linewidth of the tuning fork in the fluid and $\Delta f_{\text {vac }}$ is its linewidth due to intrinsic damping, which can be measured in vacuum, and $m_{\mathrm{vac}}$ is the effective mass of a single prong in vacuum.

As the second term in the bracket on the right hand side of Eq. 1 is usually negligible, it follows that the resonance frequency is a function of the fluid density (or in other terms - of pressure) and the linewidth is a function of both the density and the viscosity of the fluid. Therefore it is possible to use a calibrated tuning fork to infer these two quantities from its resonance characteristics, or if the temperature 
dependence of either of these is known (such as the temperature-pressure relation along the saturated vapor curve), also to measure the temperature indirectly.

A more direct temperature measurement is possible in the superfluid phases of ${ }^{3} \mathrm{He}$, where at very low temperatures, the linear dissipative force (loosely analogical to viscosity) acting on the tuning fork is determined by the concentration of thermal quasiparticles. As this concentration depends on temperature exponentially, a carefully chosen and calibrated tuning fork can work as an incredibly sensitive thermometer with a resolution of about $200 \mathrm{nK}$ at the base temperature of $500 \mu \mathrm{K}$, giving better then $10^{-3}$ resolution.

\section{TuRBulENCE DETECTION}

One of the main results obtained with the tuning forks in cryogenic fluid dynamics was the determination of the critical velocity for the transition from laminar to turbulent drag regime in both normal and superfluid ${ }^{4} \mathrm{He}$. If the drag coefficient is also evaluated as a function of velocity, additional information about what happens at the transition can be obtained as well.

We will present here the case of turbulence in ${ }^{4} \mathrm{He}$ liquids at temperatures between 4.2 and $1.3 \mathrm{~K}$, i.e., first in the range corresponding to purely normal liquid (i.e., classical viscous fluid) in the temperature range $4.2-2.17 \mathrm{~K}$, and second in superfluid ${ }^{4} \mathrm{He}$, where both its normal and superfluid components (which have independent velocity fields unless coupled due to quantized vortices) are present in nonnegligible ratios $(2.17-1.3 \mathrm{~K})$ and both can become turbulent. Other studies in ${ }^{4} \mathrm{He}$ have been performed at very low temperatures investigating (almost) pure superfluid turbulence [23].

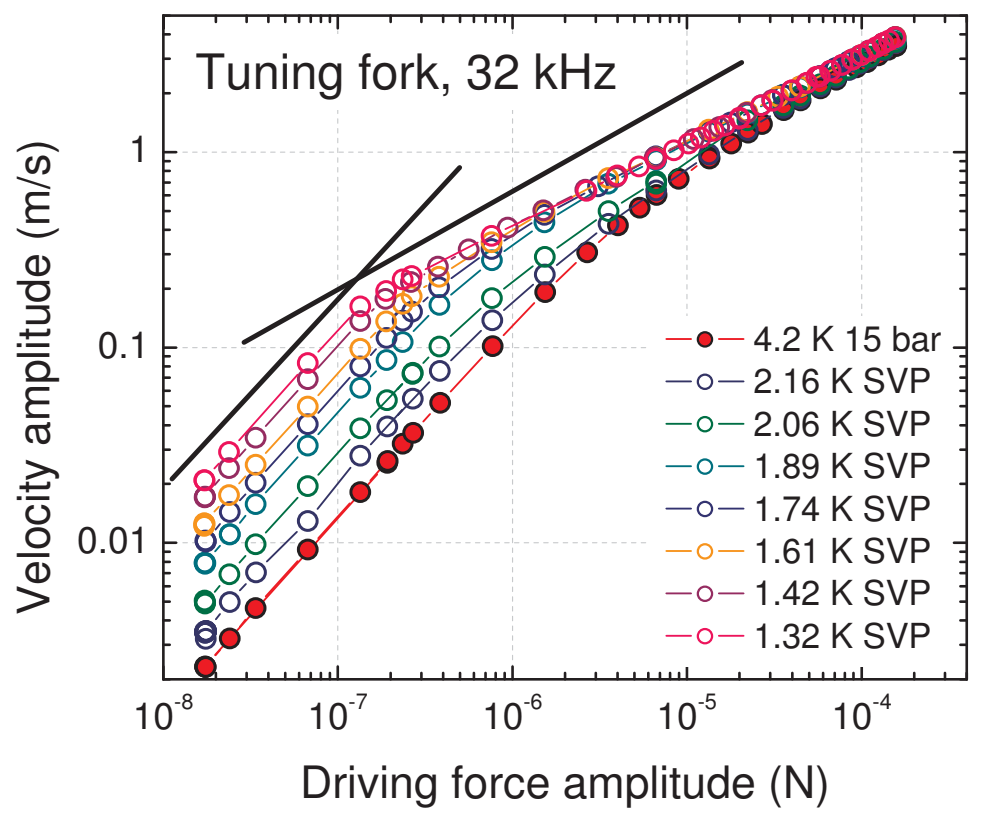

Figure 2: The force-velocity dependence measured with an opened $32 \mathrm{kHz}$ tuning fork in normal superfluid helium. The experimental data points are marked by open circles connected with solid lines in their respective series. The two straight solid lines are visual guides illustrating the transition from linear to nonlinear drag force. The dependence was found to be approximately pressure independent, but it strongly depends on the temperature of superfluid helium, i.e., on the normal fluid fraction. At the same value of velocity, the force is lower for lower temperatures as the normal fluid fraction is also lower. A transition is clearly observed in all cases as the deviation from the linear behavior seen at lower velocities. The graph also shows that the critical velocity for this transition is lower at lower temperatures. The data measured at $2.16 \mathrm{~K}$, about $0.1 \mathrm{~K}$ below the superfluid transition (taken at saturated vapor pressure), are very similar to the ones measured at $4.2 \mathrm{~K}$ at $15 \mathrm{bar}$, because the ratio of the normal component of $\mathrm{He}$ II is still very large and the fluid still behaves almost classically. At lower temperatures, the role of the superfluid component will become more important as will be better seen in Fig. 3 that shows these data plotted in terms of the drag coefficient. 
In superfluid ${ }^{3} \mathrm{He}$, which is a Fermi liquid, the situation is different - in its normal component, high viscosity prevents any turbulence from taking place under usual experimental conditions, and the only way how superfluid ${ }^{3} \mathrm{He}$ can become turbulent is via mass multiplication of quantized vortices in its superfluid component. This process is, however, associated with temperature-dependent rather than velocity-dependent critical conditions [24].

As was already mentioned above, the transition to turbulence can be detected in ${ }^{4} \mathrm{He}$ by measuring the $F(U)$ dependence of a calibrated tuning fork. Such data are plotted in Fig. 2. It is clearly shown here that a transition is observed from linear to nonlinear drag force. Additional experiments in water at room temperature (dynamically similar to the ones in normal helium) using the Baker visualization technique [25] and Kalliroscope [26] have proved that the observed change in the drag force is indeed associated with turbulence [27]. In the normal helium liquid and helium gas, which both behave as classical fluids of low kinematic viscosity, the obtained critical velocities conform to the expected scaling law of $v_{c} \propto \sqrt{\nu \omega}$, see $[28,29]$.

When the same data are plotted in terms of the drag coefficient, an unexpected feature is seen for the data taken in the superfluid, esp. at lower temperatures. At one point the drag coefficient starts to increase, then follows to reach a local maximum and decreases again to a value of order unity corresponding to fully developed turbulence, as is illustrated in Fig. 3. We believe that this feature can

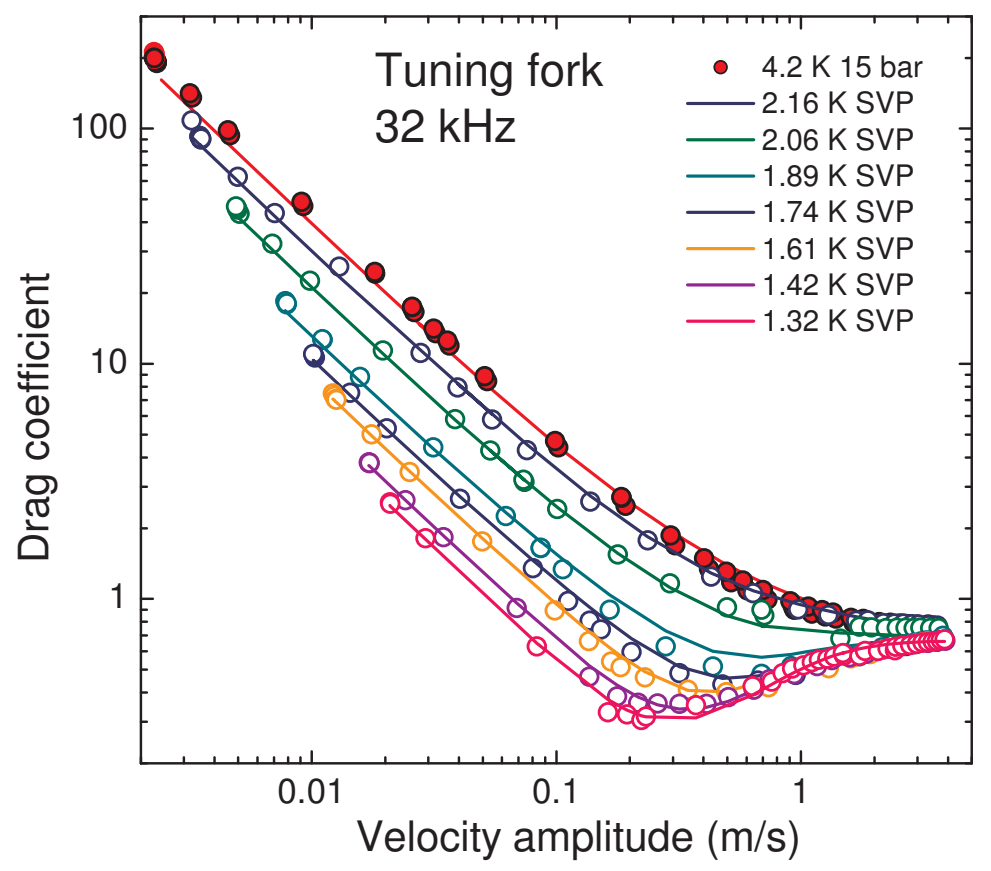

Figure 3: The drag coefficient as a function of prong velocity for various temperatures in superfluid helium. The measured data is again marked by open circles, while the solid lines correspond to fitting functions described thoroughly in [30], where a detailed analysis of the observations can be found as well. It is shown that the character of the observed transition to turbulence is very different for a classical fluid (data taken at $4.2 \mathrm{~K}$ at 15 bar) and for superfluid helium with only about $3 \%$ of the normal component (data taken at $1.32 \mathrm{~K}$ ), where a local maximum of the drag coefficient is observed before it levels off at a value of about 1 corresponding to developed turbulence. See the description in the text for more details. For the observed values of the critical velocity and the effective kinematic viscosity, please refer to [30].

help to explain the way in which the two components of superfluid helium interact during the transition and it enables us to put forward the following scenario. At the lowest velocities, the drag force is determined purely by the viscosity of the normal component, while the superfluid component exhibits potential flow. As the velocity is increased, quantized vortices begin to form in the superfluid component still without achieving fully developed turbulence, but causing coupling to the normal component. In this way, the drag coefficient increases, because additional energy is now being dissipated by the superfluid component, while dissipation occurred only in the normal component before. Finally, in fully developed 
turbulence, both components are fully coupled, behaving as a single quasi-classical fluid characterized by an effective kinematic viscosity. Viscous drag is no longer the dominant drag force, but it still functions as the eventual mechanism of energy dissipation in the normal component. An empirical mathematical model describing the two components with a varying degree of coupling has been put forward and compared with the experimental data in [30].

\section{Cavitation}

When the velocity of the tuning fork immersed in liquid helium is increased further beyond the transition to turbulence, another phenomenon becomes apparent - the tuning fork can cause cavitation near its surface. A review of cavitation in liquid $\mathrm{He}$ investigated by other means together with the standard nucleation model can be found in [31].
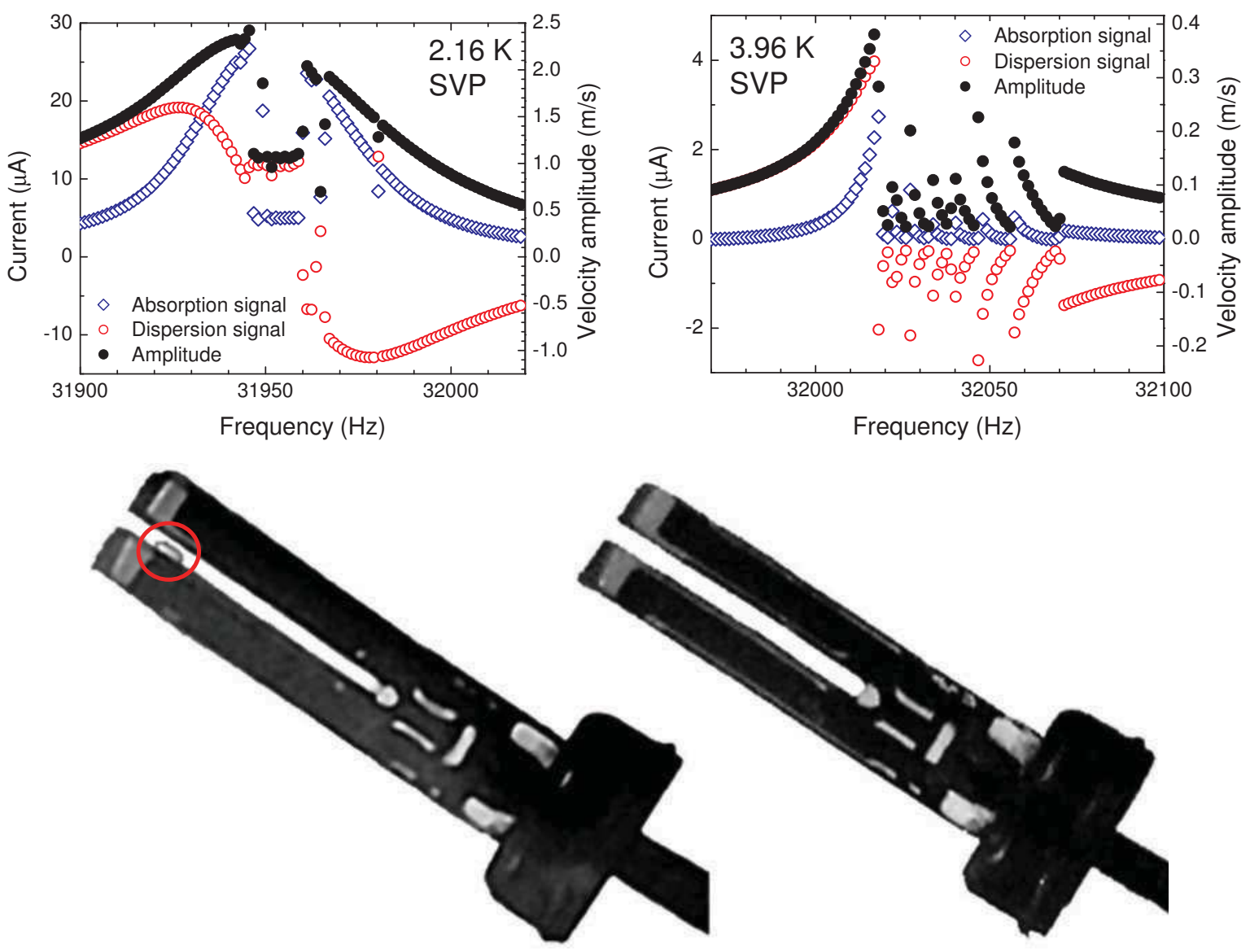

Figure 4: Illustrative graphs showing the electrical detection of cavitation in the tuning fork signal (top left and right) and a direct optical observation of a formed bubble near the tip of the tuning fork (bottom left). In the electrical measurement, it can be seen, that the formation of individual large bubbles can sometimes be detected in the signal (top right). In the photograph, the contrast on the bubble was due to reflection of artificial light on its surface and a tuning fork without bubbles is shown for comparison. (bottom right)

In our case, cavitation was observed in both normal and superfluid helium electrically (as a complete suppression of the resonance signals, as well as visually, using a digital camera to take pictures of the bubbles of gas created in our glass cryostat, Fig. see Fig. 4. Using the electrical measurements, it was possible to establish a temperature dependence of the critical velocity for cavitation to occur, which is shown in Fig. 5. The steep step-like change of the critical velocity at the superfluid transition $T=T_{\lambda}=2.17 \mathrm{~K}$ does not correspond to some fundamental physical difference between the cavitation in normal and superfluid helium, we rather believe that it was observed for another reason. We think 
that during these measurements (as well as the measurements of other groups reporting on cavitation in liquid helium), normal helium is overheated significantly, while the extremely high heat conductivity of superfluid helium (at least six orders of magnitude higher by comparison) is sufficient to maintain an approximately constant temperature in the spot where cavitation occurs. Therefore an incorrect value of the critical velocity is obtained for normal helium, as the nucleation line in the phase diagram is not crossed in the direction of the pressure axis, but rather at an arbitrary angle to it, corresponding to the fact that both underpressurizing and heating occur simultaneously. A more detailed account of these results is given in $[32,33]$.

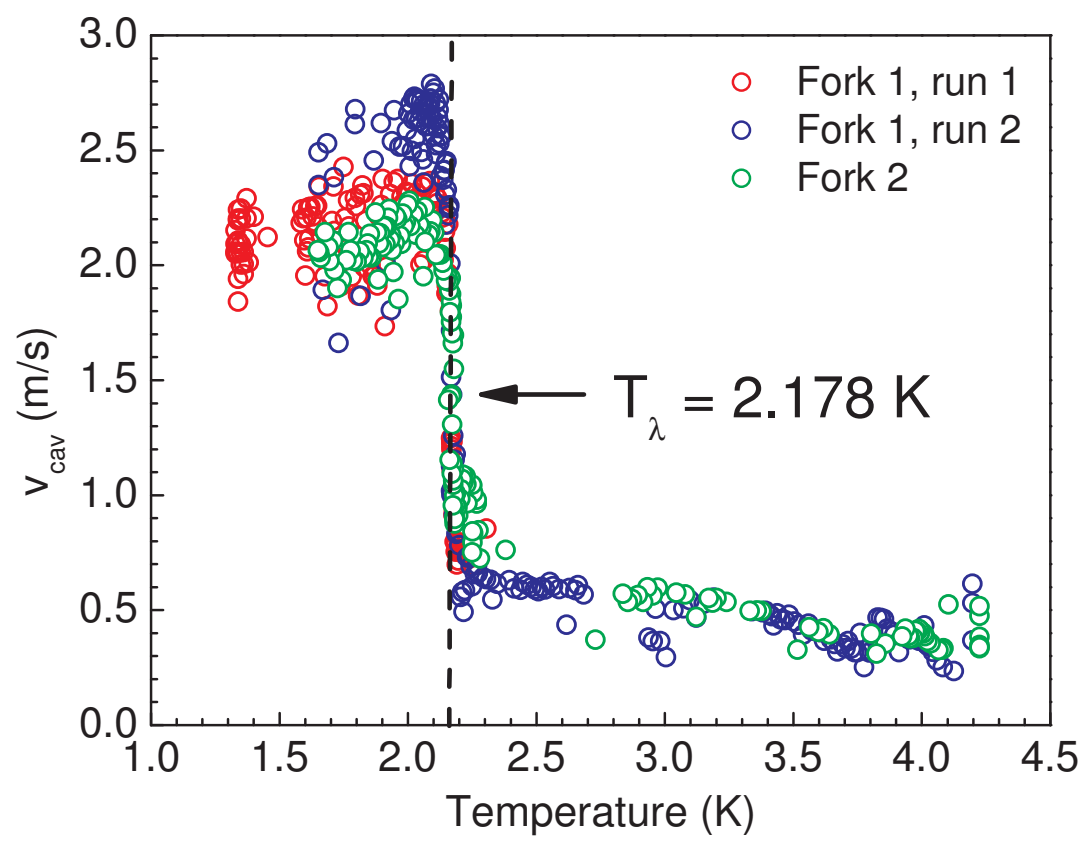

Figure 5: Critical velocity for cavitation measured with $32 \mathrm{kHz}$ tuning forks inside their cans plotted versus temperature. We believe that the step measured at the superfluid transition is not an intrinsic property of superfluid helium but is observed because the values in normal helium above $2.17 \mathrm{~K}$ are measured in a locally overheated fluid, which makes cavitation easier, see the text for more details.

Similar measurements were repeated in a pressure cell at $4.2 \mathrm{~K}$ and the resulting overpressure dependence of the critical velocity is presented in Fig. 6. In thermodynamical equilibrium, cavitation should occur at a certain (under-)pressure for each value of temperature. Here, the actual pressure at the spot where the bubble is formed, is reduced due to the flow velocity of liquid helium past the oscillating tuning fork. Therefore, the quadratic character of the observed velocity versus overpressure dependence suggests that to first approximation, the pressure reduction due to a finite flow velocity can be roughly expressed from the Bernoulli equation, even though the flow past the tuning fork is already turbulent. Fitting the Bernoulli equation to the data gives us the estimates of the relevant flow enhancement factors past the sharp corners of the tuning forkas they are shown in Fig. 6.

\section{ACOUSTIC PROPERTIES OF TUNING FORKS}

Most of the investigations carried out with the tuning forks neglected acoustic emission entirely. It is mentioned for the first time in [34]. Lately, it was found that it might be of considerable importance when dealing with the quartz tuning forks and can be even used to measure various physical quantities such as speed of sound in ${ }^{3} \mathrm{He}{ }^{4} \mathrm{He}$ mixtures [35].

As is the case with the common musical tuning forks, even the tiny forks used in cryogenic experiments do emit sound, which contributes to the overall damping they experience. The fact is that in most cases, the losses due to acoustic emission for the standard tuning forks are negligible when compared to viscous dissipation, and that is also the reason why it was often simply omitted, occasionally even without sufficient justification. However, two important exceptions regarding the negligibility of acoustic 


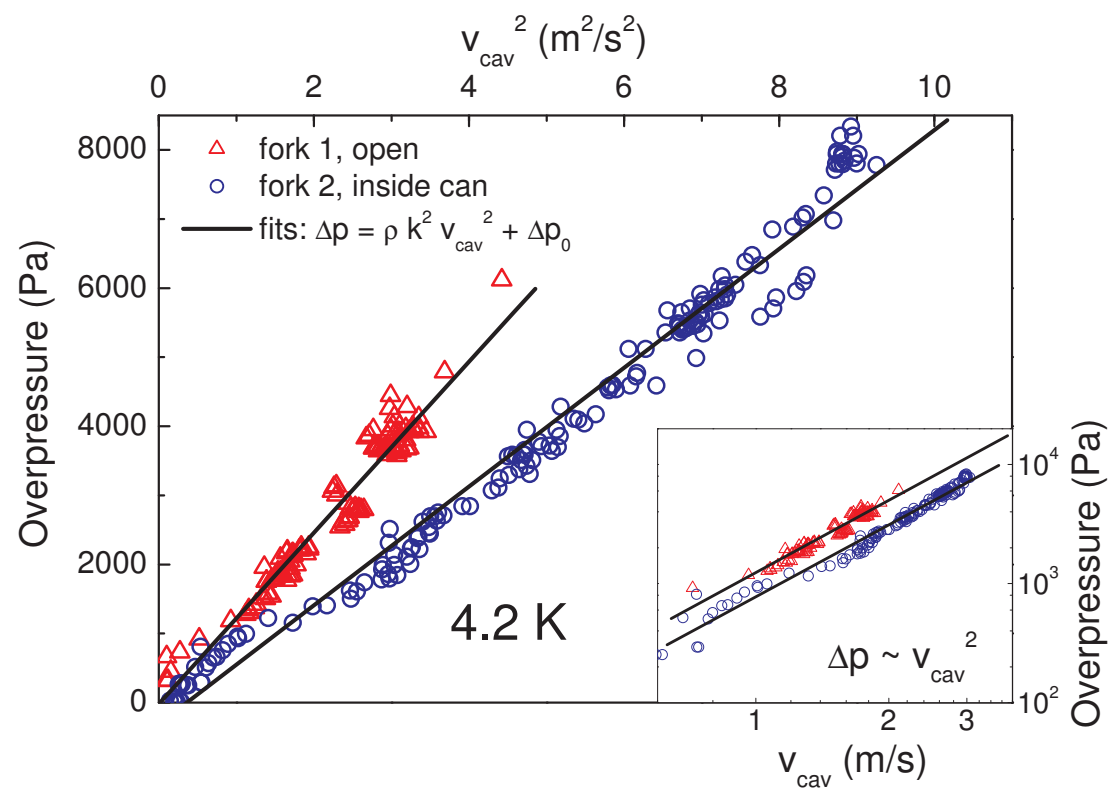

Figure 6: The square of the critical cavitation velocity as a function of overpressure is shown in linear axes in the main window. The critical velocity itself is shown in the logarithmic inset that also proves the quadratic dependence of the velocity on overpressure. This dependence enables us to estimate the flow enhancement factors, $k$, past the sharp corners of the tuning fork if we assume the description by the Bernoulli equation to be valid at least approximately. The values of $k$ corresponding to the fits are $k=3.1$ for the open fork and $k=2.6$ for the fork inside its can.

emission exist. First, as the emission power is highly frequency dependent $\left(f^{5}\right.$ or $f^{6}$ depending on whether we prefer to use a $2 \mathrm{D}$ or $3 \mathrm{D}$ description of the acoustic fields), it is evident that if we replace the standard forks oscillating at $32 \mathrm{kHz}$ by higher frequency varieties (such as $77 \mathrm{kHz}$ and $100 \mathrm{kHz}$ forks) the acoustic emission will become several orders of magnitude stronger. The second exception comes into play when operating even the standard forks at very low temperatures in ${ }^{4} \mathrm{He}$. As the ratio of the normal component decreases rapidly with decreasing temperature, so does the viscous drag force (the superfluid component being inviscid) and eventually, acoustic emission (or phonon emission, if the reader prefers) will become the dominant dissipative process. A detailed treatment of the acoustic emission by tuning forks including several analytical models will be available in [36].

Presently, it is also believed that acoustic emission may be partly responsible for the observed interaction between two different tuning forks placed inside the same container, which was previously exclusively attributed to quantized vortices, see [37]. However, while these experiments do not pose any severe technical problems, it is extremely difficult to perform systematic investigations and to interpret the measured data and at this instant, the achieved understanding is far from complete.

\section{Conclusions}

During the last decade, the quartz tuning fork has become a popular experimental tool within the cryogenic fluid dynamics community to study flows both in classical and superfluid phases of both helium isotopes. In ${ }^{4} \mathrm{He}$, it was and still is successfully being used to study the transition to turbulence under various conditions, it helped to provide an insight into cavitation in normal and superfluid helium and recently its acoustic properties and their effects are being studied as well. In ${ }^{3} \mathrm{He}$, it is commonly used as an extremely sensitive secondary thermometer and also as a handy means of generating and/or detecting quantized vortices (thanks to the phenomenon known as Andreev reflection). While the tuning fork has already yielded significant results, many challenges still remain open for further investigation, for example the detailed description of the emission of first and second sound and perhaps even more importantly, the exact nature of the interaction between multiple tuning forks immersed in the same volume of fluid. 
As very sensitive detectors, quartz tuning forks of varying sizes and resonant frequencies have also found use in other areas of interest. They are becoming common tools in viscosity measurements, especially for various oils and other electrically insulating fluids, as otherwise, they would have to be coated carefully by a thin insulating layer in order to be used, e.g., in water or alcohol. Similarly, they can be used in flow measurement applications, providing an excellent solution where the size of the detector is an important issue, albeit at a reduced accuracy. Altogether, it can be taken for granted that the quartz tuning fork will find many more uses both in scientific research and in industry, and certainly deserves to receive the continued attention of the involved communities.

\section{REFERENCES}

[1] L Skrbek, W F Vinen, The use of vibrating structures in the study of quantum turbulence, Progress in Low Temperature Physics, vol.XVI, Chapter 4, ed. by M. Tsubota, W.P. Halperin, Elsevier, Amsterdam, 2009

[2] D R Tilley, J Tilley, Superfluidity and superconductivity, Adam Hilder Ltd , Bristol 1986

[3] W F Vinen, J J Niemela, Quantum turbulence, J. Low Temp. Phys. 128, 167, 2002

[4] L Skrbek, K R Sreenivasan, Developed quantum turbulence and its decay, to appear in Phys. Fluids.

[5] R J Donnelly, K R Sreenivasan, Flow at ultra-high Reynolds and Rayleigh numbers, Springer, New York, 1998

[6] L Skrbek, J J Niemela, R J Donnelly, Turbulent flows at cryogenic temperatures: A new frontier, Journal of Physics: Condensed Matter 11, 7761, 1999

[7] J J Niemela, K R Sreenivasan, The use of cryogenic helium for classical turbulence: Promises and hurdles, J. Low Temp. Phys. 143, 163, 2006

[8] R J Donnelly, C F Barenghi, The observed properties of liquid helium at the saturated vapor pressure, J. Phys. Chem. Data 27, 1217, 1998

[9] J J Niemela, L Skrbek, K R Sreenivasan, R J Donnelly, Turbulent Convection at Very High Rayleigh Numbers Nature 404, 837, 2000

[10] P-E Roche, F Gauthier, R Kaiser, J Salort, On the triggering of the ultimate regime of convection, New Journal of Physics, 12, 085014, 2010

[11] P Urban, P Hanzelka, T Králík, V Musilová, L Skrbek, A Srnka, Helium cryostat for exprimental study of natural turbulent convection, Review of Scientific Instruments 81, 085103, 2010

[12] J Maurer, P Tabeling, Local investigation of superfluid turbulence, Europhys. Lett. 43, 29, 1998

[13] P-E Roche, P Diribarne, T Didelot, O Francais, L Rousseau, H Willaime, Vortex density spectrum of quantum turbulence, Europhys. Lett. 77, 66002, 2007

[14] H Yano et al, Observation of laminar and turbulent flow in superfluid He-4 using a vibrating wire, J Low Temp Phys 138, 561, 2005

[15] D Charalambous et al , Experimental investigation of the dynamics of a vibrating grid in superfluid He-4 over a range of temperatures and pressures, Phys Rev E 74, 036307, 2006

[16] D I Bradley et al, Emission of discrete vortex rings by a vibrating grid in superfluid He-3-B: A precursor to quantum turbulence, Phys Rev Lett 95, 035302, 2005

[17] M Niemetz, W Schoepe, Stability of laminar and turbulent flow of superfluid He-4 at mK temperatures around an oscillating microsphere, J Low Temp Phys 135, 447, 2004 
[18] J Luzuriaga, Measurements in the laminar and turbulent regime of superfluid ${ }^{4} \mathrm{He}$ by means of an oscillating sphere, J. Low Temp. Phys. 108, 267, 1997

[19] D Schmoranzer, L Skrbek, The use of vibrating quartz forks in cryogenic helium research - On their ability to detect an externally applied flow in superfluid ${ }^{4} \mathrm{He}$, J. Phys. Conf. Series 150, 012048, 2009

[20] R Blaauwgeers et al , Quartz tuning fork: Thermometer, pressure- and viscometer for helium liquids, J Low Temp Physics 146, 537, 2007

[21] M Blažková, et al, Vibrating quartz fork - a tool for cryogenic helium research, Journal of Low Temp. Physics 150, 525, 2008

[22] Lancaster optical calibration, to be published

[23] D I Bradley, et al, Transition to turbulence for a quartz tuning fork in superfluid ${ }^{4} \mathrm{He}$, J Low Temp Phys, 103, 116, 2009

[24] A P Finne,et al, Observation of an intrinsic velocity-independent criterion for superfluid turbulence, Nature 424, 1022, 2003

[25] D J Baker, A technique for precise measurement of small fluid velocities, J. Fluid Mechanics. 26, 573,1966

[26] A rheoscopic fluid, see www.kalliroscope.com

[27] D Schmoranzer, M Králová, V Pilcová, V F Vinen, L Skrbek, Experiments relating to the flow induced by a vibrating quartz tuning fork and similar structures in a classical fluid, Phys. Rev. E 81, 066316, 2010

[28] M Blažková, D Schmoranzer, L Skrbek, emphTransition from laminar to turbulent drag in flow due to a vibrating quartz fork, Phys. Rev. E 75, 025302, 2007

[29] M Blažková, M Človečko, L Skrbek, P Skyba, Quantum turbulence generated and detected by a vibrating quartz fork, J. Low Temp. Physics 148, 305, 2007

[30] M Blažková et al, The generation of turbulence by vibrating forks and other structures in superfluid ${ }^{4} \mathrm{He}$, Phys. Rev. B, 79, 054522, 2009

[31] S. Balibar, Nucleation in quantum liquids , J. Low Temp. Phys. 129, 363, 2002

[32] M Blažková, T V Chagovets, M Rotter, D Schmoranzer, L Skrbek, emphCavitation in liquid helium observed in a flow due to a vibrating quartz fork, J. Low Temp. Phys., 150, 194, 2008

[33] M Blažková, D Schmoranzer, L Skrbek, On Cavitation in Liquid Helium in a Flow Due to a Vibrating Quartz Fork, Low Temp Phys, 34, 298, 2008

[34] D O Clubb, O V L Buu, R M Bowley, R Nyman, J R Owers-Bradley, Quartz tuning fork viscometers for helium liquids, J. Low Temp. Phys., 136, 1, 2004

[35] E M Pentti, J T Tuoriniemi, A J Salmela, A P Sebedash, Quartz tuning fork in helium, J. Low Temp. Phys., 150, 555, 2008

[36] D Schmoranzer, M LaMantia, G Sheshin, I Gritsenko, A Zadorozhko, M Rotter, L Skrbek, Acoustic emission by quartz tuning forks in cryogenic ${ }^{4} \mathrm{He}$ fluids, to appear in J. Low Temp. Phys.

[37] D Garg, V B Efimov, M Giltrow, P V E McClintock, L Skrbek, V F Vinen, Tuning forks in superfluid ${ }^{4} \mathrm{He}$ at a very low temperature: anomalous damping, and fork-fork interactions, submitted to Europhys. Lett. 